\title{
Análisis del tratamiento y nivel de contaminantes por lámparas fluorescentes en El Salvador
}

\section{Analysis on the treatment and level of contaminants in fluorescent lamps in El Salvador}

\author{
José Remberto Miranda-Mejía' \\ josé.miranda@utec.edu.sv \\ Samuel Martínez-Gómez² \\ John Figerald Kenedy Hernández-Miranda ${ }^{3}$
}

Investigadores

Recibido: 11/05/2015 - Aceptado: 08/06/2015

\section{Resumen}

El estudio consiste en analizar los tratamientos actuales que se aplican a los desechos que generan las lámparas fluorescentes, realizar propuesta de la disposición final de las lámparas e identificar los materiales reutilizables. El mercurio puede cambiar de forma (principalmente por metabolismo microbiano) y convertirse en metilmercurio, que tiene la capacidad de acumularse en organismos (bioacumulación) y concentrarse en las cadenas alimentarias (biomagnificación), especialmente en la cadena alimentaria acuática (peces y mamíferos marinos). Se realizó un estudio empírico con metodología cuantitativa. También como parte el estudio descriptivo mediante un código arbitrario de observación tipo estructurada. Se evaluaron 32 lugares, de los cuales uno es del departa-

\section{Abstract}

The study consists of [the following elements:] the analysis of the current waste treatment of fluorescent lamps, the proposal for proper final disposal of the lamps, and the identification of reusable materials. Mercury can change shape (primarily by microbial metabolism) to become methylmercury, which has the capacity to accumulate in organisms (bioaccumulation) and concentrate in food chains (fish and marine mammals), especially in the aquatic food chain (fish and marine mammals). An empirical study was conducted following a quantitative methodology. It was also part of a descriptive study by means of an arbitrary observation code of a structuredtype. Of the 32 sites evaluated, one from the department of San Salvador and three from the department of La

Investigador y docente de la Universidad Tecnológica de El Salvador, ingeniero industrial, con posgrado en investigación científica, autor del libro Mejores prácticas en preparación de alimentos en la micro y pequeña empresa y del estudio "Análisis al tratamiento actual de las lámparas fluorescentes, nivel de contaminantes y disposición final", publicaciones en revista entorno y participaciones en congresos nacionales. Correo electrónico jose.miranda@utec.edu.sv.

Investigador y docente horas-clase de la Universidad Tecnológica de El Salvador es ingeniero industrial con maestría en Investigación de Operaciones (modelos de decisión), autor del libro Análisis al tratamiento actual de las lámparas fluorescentes, nivel de contaminantes y disposición final. correo electrónico samuelmg050@hotmail.com.

3 Ingeniero industrial e investigador, inspector de seguridad ocupacional del Ministerio de Trabajo y Previsión Social, autor del libro Análisis al tratamiento actual de las lámparas fluorescentes, nivel de contaminantes y disposición final. Correo electrónico kenedy.mtps@gmail.com 
mento de San Salvador y tres del departamento de La Libertad, en los que se encontró emisiones de mercurio a la atmósfera.

\section{Palabras clave}

Mercurio, bioacumulación, biomagnificación.
Libertad showed the presence of mercury emissions in the atmosphere.

\section{Keywords}

Mercury, bioaccumulation, biomagnification

\section{Historia de lámparas fluorescentes}

A finales del siglo 19, Thomas Alva Edison desarrolló su versión de lámpara fluorescente eléctrica, la cual catalogó como ineficiente; a la vez que fue difícil de reproducir en serie. Luego, en la década de 1920, la ineficiencia de las lámparas continuó debido a la falta del fósforo apropiado y de una fuente de radiación ultravioleta para integrársela. Fue hasta en la década de los 30, en los que se desarrollaron fósforos eficientes para las lámparas de vapor de mercurio, que resultó en una fuente eficiente de radiación ultravioleta. Las lámparas fluorescentes también se colocaron como una novedad en la Feria Mundial de Chicago en 1938, y fue en Nueva York donde estuvieron disponibles para el público en abril de 1938 (Flavin, 2008). Se estima que desde 1940 a la fecha se ha importado y ocupado la lámpara fluorescente en El Salvador como fuente de luz artificial. La forma en que las lámparas fluorescentes producen luz artificial es descrita a continuación.

\section{Formas de producción de la radiación luminosa}

Todas las fuentes de luz artificial implican la conversión de alguna forma de energía en radiación electromagnética. Este es un proceso físico que se da a nivel atómico; la excitación y subsiguiente desexcitación de átomos o moléculas es el proceso más empleado para la generación de luz en las fuentes artificiales (O'Donell, Sandoval, \& Paukste, 2006).

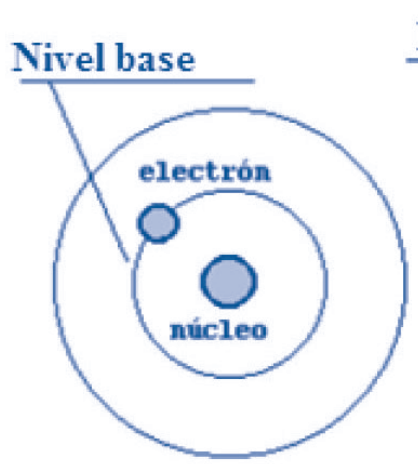

(a)

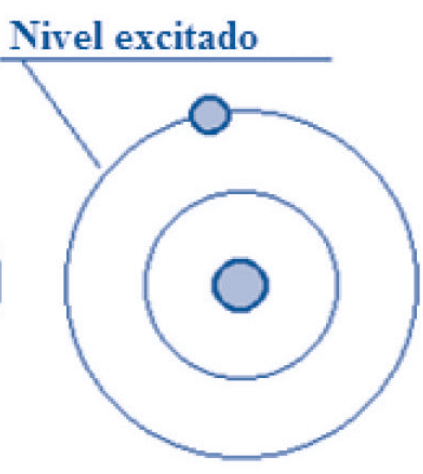

(b) Átomo normal

\section{Transición radiante}

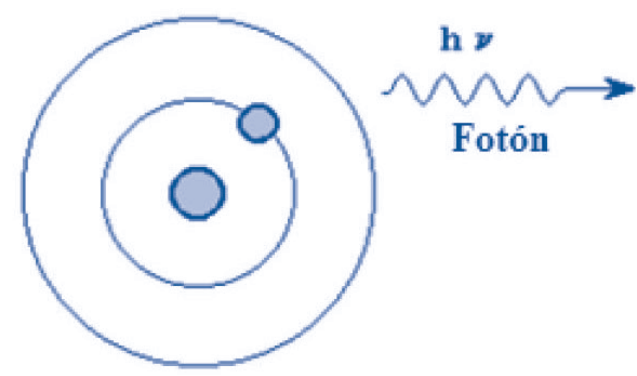

(c)

Emisión de radiación

Figura 1.

Excitación atómica y emisión espontanea de radiación

(O'Donell, Sandoval, \& Paukste, 2006). 
En la figura 1 se muestra, en la parte (a), un átomo consistente en un núcleo con un electrón girando alrededor de él en una órbita estable; el átomo es excitado mediante el choque con alguna otra partícula, de modo que el electrón se mueve a otra órbita con un nivel de energía mayor, como se muestra en (b); luego, el electrón en estado excitado caerá espontáneamente a su órbita estable, como en la parte (c) de la figura, devolviendo en esta desexcitación el exceso de energía en forma de un fotón o unidad de luz. Este fenómeno se conoce como emisión de radiación (O'Donell, Sandoval, \& Paukste, 2006). En el siguiente párrafo se describe el funcionamiento de las lámparas fluorescentes.

\section{Lámparas de descarga}

Se denominan lámparas de descarga a todas aquellas fuentes luminosas cuyo principio de funcionamiento consiste en generar luz mediante una descarga eléctrica, producida entre dos electrodos situados en el interior de un tubo lleno de gas; a diferencia de las lámparas incandescentes en las que la luz emitida se debe a las altas temperaturas alcanzadas en el filamento. Las lámparas de descarga se pueden clasificar en distintos tipos, según el gas utilizado y la presión a la que se encuentre en el interior del tubo de descarga (ELT, 2006).

Las lámparas fluorescentes son activadas por vapor de mercurio a baja presión $(0.8 \mathrm{~Pa})$; son fuentes luminosas a consecuencia de una descarga eléctrica en atmósfera de vapor de mercurio a baja presión, en las que la luz se genera por el fenómeno de la fluorescencia. Este consiste en que determinadas sustancias luminiscentes, al ser excitadas por la radiación ultravioleta invisible del vapor de mercurio a baja presión, transforman esta radiación en otra radiación visible. La lámpara fluorescente normal consta de un tubo de vidrio de diámetro y longitud variada, según la potencia, recubierto internamente de una capa de sustancia fluorescente (Inti, 2014). Los diferentes tipos de lámparas fluorescentes son las tubulares, compactas, de alta presión y de halogenuros metálicos. A continuación se describen dos de las más usadas en El Salvador.

\section{Lámparas fluorescentes tubulares}

Se denominan lámparas fluorescentes tubulares o lineales a aquellas que presentan el tubo de descarga en forma rectilínea, aunque también se encuentran dentro de esta denominación las lámparas en formas de $U$ o circulares. Los diámetros de dichos tubos son 7, 16, 26 (T8) y 38 mm (T12); su eficacia lumínica (cantidad de luz por vatio de potencia) se encuentra entre los 50 y $100 \mathrm{Im} / \mathrm{W}$, dependiendo del tipo de lámpara (Cook, 1998; General Electric, 2014).

\section{Lámparas fluorescentes compactas}

Se denominan lámparas fluorescentes compactas a las formadas por un tubo estrecho, de entre 10 y $16 \mathrm{~mm}$ de diámetro, doblado en forma de $U$, al que se le han unido los extremos para obtener dimensiones reducidas. Reducir las dimensiones de las lámparas a tamaños semejantes a las bombillas incandescentes, 0 algo mayores, permite que sean utilizadas en alojamientos donde las lámparas lineales de potencias iguales no caben (Martínez, 1998). Su vida media y útil son algo inferiores a la de las lámparas lineales.

\section{Funcionamiento de lámpara fluorescente}

Conectadalalámparaensu circuitoeléctricocorrespondiente, la corriente que atraviesa los cátodos los calienta y emiten electrones. Una vez que se ha establecido en el interior de la lámpara la nube de electrones susceptibles de movimiento, se aplica una sobretensión entre los extremos de la lámpara (Martínez, 1998).

En los extremos del tubo se encuentran los cátodos de wolframio impregnados de una pasta emisora de electrones. Los electrones pasan de un cátodo a otro a través de la atmósfera de argón del interior del tubo, iniciándose la descarga. El calor producido por la descarga evapora rápidamente el mercurio, por lo que la descarga se mantiene en una atmósfera de mayor conductividad, mezcla del gas argón y del vapor de mercurio. Los electrones, en su recorrido de un cátodo al otro, chocan con los átomos de mercurio, desprendiendo una energía que se transforma en radiación ultravioleta invisible capaz de excitar la sustancia fluorescente de la capa que recubre interiormente el tubo, convirtiéndose en luz visible (Martínez, 1998).

\section{Radiación ultravioleta de las lámparas fluorescentes}

Las lámparas fluorescentes no deben emitir ninguna radiación que pueda resultar peligrosa para el hombre, ya sea 
de inmediato o a largo plazo. En el caso de algunas descargas de gases, principalmente las de vapor de mercurio, poseen por naturaleza un porcentaje de radiación ultravioleta. Por esta misma razón las lámparas fluorescentes llevan mercurio, que les permite generar esa radiación para activar el polvo fluorescente de las lámparas (Indalux, 2002).

\section{Riesgos con la radiación ultravioleta (UV) emitida por las lámparas fluorescentes compactas LFC}

La exposición a la luz brillante y a la de alta temperatura de color se debe evitar en la noche. Las propiedades de las lámparas fluorescentes compactas (LFC) que pudieran suponer un riesgo de salud son la luz ultravioleta y la azul emitidas en las longitudes de ondas cortas (por debajo de $530 \mathrm{~nm}$ ), ya que pueden retrasar el ritmo circadiano de los mamíferos, y esto puede reducir la secreción de melatonina. Bloquear la luz azul para la reducción del riesgo en las personas que ya padecen enfermedades de la piel es necesario, porque pueden agravar sus síntomas. La disminución en la producción de melatonina en humanos y animales causada por la iluminación ambiental y por la de longitud de onda, especialmente corta (entre 470 y $525 \mathrm{~nm}$ ), se ha demostrado que se asocia a un mayor riesgo de cáncer (Kayumov, Lowe, Rahman, Casper, \& Shapiro, 2007).

En ensayos realizados a una reducida cantidad de lámparas para medir su emisión de radiación UV, estas presentaron picos de emisión UV en 365 nm, por lo que esto sería riesgoso para la salud cuando se usa muy cerca del usuario por tiempo prolongado. Se puede mencionar que en el caso de otra de las lámparas el pico fue de $365 \mathrm{~nm}$, que es mayor que el de $485 \mathrm{~nm}$ (azul), tanto para lámparas nuevas como para las que ya tienen 1.000 horas de uso (Echazú \& Cadena, 2012).

En un experimento con once participantes masculinos jóvenes sanos (edad media $23.5 \pm 1.5$ años), los niveles de melatonina salivales se midieron bajo tenue luz ( $<5$ lux), luz brillante (800 lux) y luz filtrada (800 lux) con intervalos de una hora entre 2000 y 0800 h, durante tres días no consecutivos en dos semanas, utilizando lentes de filtro óptico, se demostró que nueve conservaron los niveles de melatonina en la luz filtrada, resultado similar a su perfil de secreción en luz tenue. Con luz filtrada, los participantes tuvieron una cantidad relativa media de melatonina de 91,2 $(P>0,05)$ (Kayumov et al., 2007). Al experimentar con luz brillante sin filtrar, esta exposición suprimió drásticamente la producción de melatonina con una cantidad relativa media de la melatonina de $25,4(p<0,05)$. Una solución práctica y rentable para evitar el aumento de tasas de tumores malignos en trabajadores expuestos a luz de longitud de onda baja ha sido el usar lentes bloqueadores para que no llegue a la retina (Kayumov et al., 2007).

\section{Importaciones anuales de lámparas fluorescentes}

En El Salvador se venden varios tipos de luminarias que contienen mercurio, entre las que se encuentran las fluorescentes lineales, fluorescentes circulares, compactas fluorescentes, de sodio de alta presión y de mercurio.

Figura 2.

Importaciones anuales de lámparas fluorescentes lineales de los últimos trece años (Banco Central de Reserva \& Ministerio de Hacienda, Direccion General de Aduanas, 2014)

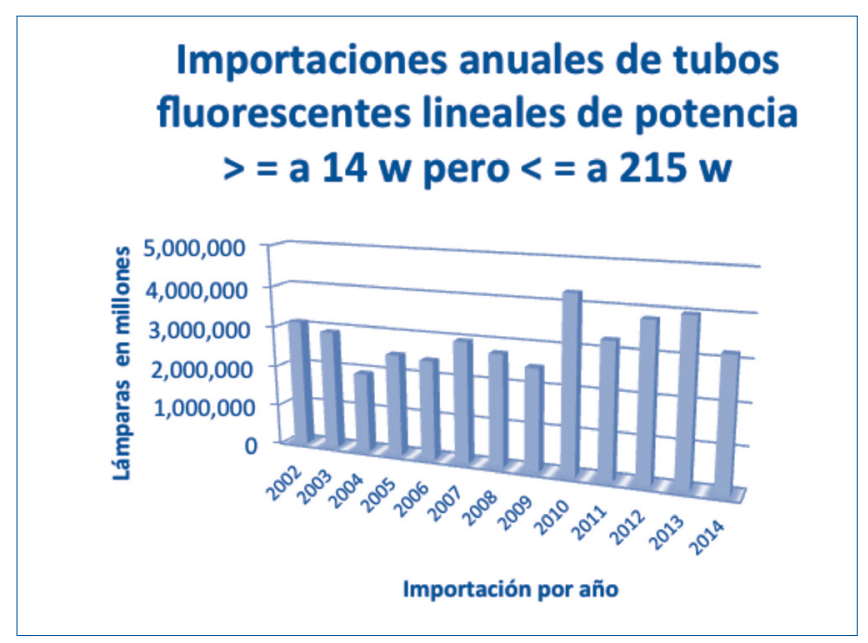

La figura 2 muestra las importaciones anuales de lámparas fluorescentes lineales a El Salvador durante los últimos 13 años, con una media de 3.053.896, en las que la del 2010 fue la mayor, con 4.291.211 unidades. 
Figura 3.

Importaciones de lámparas fluorescentes circulares de los últimos once años (Banco Central de Reserva \& Ministerio de Hacienda-Direccion General de Aduanas, 2014)

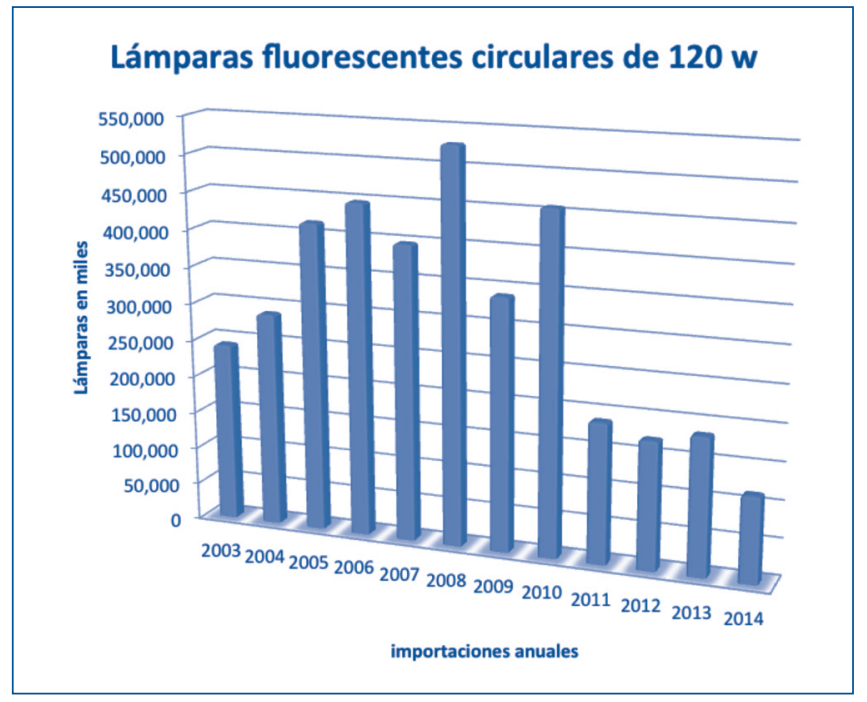

En la figura 3 se muestran las importaciones anuales de los últimos trece años de lámparas fluorescentes tubulares, que en el año 2008 alcanzaron la cantidad de 530.000 unidades. Estas se suman a otros tipos de lámparas que contienen mercurio, a las que no se les ha dado tratamiento para extraerle el mercurio.

\section{Emisiones de mercurio por lámparas fluorescentes}

Las lámparas fluorescentes inservibles fueron declaradas por la Agencia de Protección Ambiental de EE. UU. (EPA) como desechos peligrosos que deberían tener un manejo más riguroso, debido al contenido de mercurio y otros 17 elementos peligrosos según la Ley de Conservación y Recuperación de Recursos. Debido a las características de este metal es que estos desechos son nocivos, ya que el elemento mercurio es el más tóxico de todos los metales; no es esencial para ningún proceso metabólico y es bioacumulable en la mayoría de los seres vivos (UGR, 2013).

El proceso de contaminación ambiental por lámparas inicia cuando estas son rotas, al depositarlas de forma inadecuada en los basureros o al ser recolectadas por camiones no aptos para el transporte de este tipo de desechos; y en los rellenos sanitarios, cuando los lixiviados que se generan están en contacto con lámparas fluorescentes, que contaminan Ios lixiviados con mercurio (Angulo \& Romero, 2006). Estos, al infiltrarse en el suelo, son capaces de alcanzar aguas subterráneas, las cuales llegan a cursos de aguas superficiales; y luego el agua es ocupada para usos múltiples (Angulo \& Romero, 2006).

\section{El mercurio en el medio ambiente}

“El mercurio metálico se encuentra ampliamente distribuido en la naturaleza en concentraciones muy bajas. En la tierra no contaminada, las concentraciones de mercurio varían de 30 a 500 partes por millón de millones (partes por billón, $\mathrm{ppb})$, con un promedio de aproximadamente $100 \mathrm{ppb}$ (EPA, 1997). Para la mayoría de las rocas, el contenido de mercurio varía de 10.000 a 20.000 ppb. Salvo condiciones geológicas especiales o donde las fuentes antropogénicas llevan a aumentos, las aguas superficiales en general contienen menos de $0.1 \mathrm{ppb}$ de mercurio total; y el agua de mar, promedios de 0.1 a 1.2 ppb de mercurio" (EPA, 1997).

Este elemento se encuentra actualmente en diversos medios y alimentos (especialmente en el pescado), en todas partes del mundo, a niveles que causan efectos adversos a los seres humanos y en la vida silvestre. La actividad del hombre ha generalizado los casos de exposición, y las prácticas del pasado han dejado un legado de mercurio en vertederos, los desechos de la minería y los emplazamientos, suelos y sedimentos industriales contaminados. Hasta las regiones donde se registran emisiones mínimas de mercurio, como la del Ártico, se han visto afectadas debido al transporte transcontinental y mundial del mercurio (OMS, 2013).

\section{Ciclo biogeoquímico}

El mercurio tiene un ciclo, en el ambiente, en el que continuamente recircula en diferentes medios en los que se dan las reacciones de metilación, ya que tiene tres estados de oxidación $(0,+1,+2)$ por medio de procesos biológicos y la bioacumulación en los diferentes organismos vivos a través del aire, el agua y los alimentos (O'Neill, 1993, 1998).

El mercurio atmosférico puede incorporarse fácilmente a los sistemas orgánicos y al agua por deposición (UGR, 2013). El destino, características del transporte y de toxicidad del 
mercurio pueden ser significativamente afectados por la fase física y de la especiación química. En consecuencia, es importante que estos parámetros se tomen en cuenta cuando se estudia el ciclo del mercurio en el medio ambiente (Constantinou, Gerath, Mitchell, \& Seigneur, 1995).

Cuando el mercurio es depositado en el ambiente puede cambiar de forma inorgánica a orgánica (principalmente por metabolismo microbiano) y convertirse en metilmercurio $\left(\mathrm{CH}_{3}\right)$, que tiene la capacidad de acumularse en organismos (bioacumulación) y concentrarse en las cadenas alimentarias (biomagnificación), especialmente en la cadena alimentaria acuática (peces y mamíferos marinos). Casi todo el mercurio que se encuentra en los peces es metilmercurio (UGR, 2013).

El mercurio es una sustancia química cuyo comportamiento es particularmente sensible a las condiciones ambientales y sobre todo a las características del medio acuático (Constantinou, et al., 1995). En el medio acuático las concentraciones de mercurio han aumentado considerablemente como resultado de actividades antropogénicas. Las mediciones oceanográficas de mercurio disuelto total y los parámetros relacionados de varias expediciones al Atlántico, Pacífico Sur y Ártico. Se encontró que aguas profundas del Atlántico Norte y la mayoría de las aguas intermedias están anómalamente enriquecidas en mercurio en relación con las aguas profundas del Atlántico Sur y Pacífico Sur, probablemente como resultado de la incorporación de mercurio antropogénico (Lamborg, et al., 2014). Esto contradice el estudio "Localización y estimación de las emisiones atmosféricas provenientes del mercurio y compuestos de mercurio" realizado por EPA en 1997, que se mencionó anteriormente.

Se ha calculado que la cantidad total de mercurio antropogénico presente en los océanos del mundo puede ser de $80 \pm 290$ millones de moles, de los que casi dos tercios están en aguas de menos de mil metros de profundidad. Estos hallazgos sugieren que las perturbaciones antropogénicas del ciclo global del mercurio han llevado a un aproximado de $150 \%$ de aumento en la cantidad de mercurio en las aguas de la termoclina, y se ha triplicado el contenido de mercurio de las aguas superficiales de los mares en comparación con condiciones pre-antropogénicas (Lamborg, et al., 2014).

\section{El mercurio en el hombre y la naturaleza}

\section{Usos del mercurio}

Según el grado de contaminación local con mercurio, la ingesta total de mercurio por el agua y el aire puede aportar otras cantidades sustanciales. El uso de mercurio con ciertos fines religiosos, culturales y rituales; su uso en algunas medicinas tradicionales y en el hogar y el medio ambiente de trabajo puede dar lugar a aumentos sustanciales de la exposición humana (Montenegro \& Nicolalde, 2012; PNUMA, 2013). También se produce exposición por el uso de vacunas y otros productos farmacéuticos que contienen preservantes de mercurio (como el timerosal o tiomersal). Se ha notificado niveles elevados de mercurio elemental en el entorno de trabajo de plantas de cloro álcali, minas de mercurio, fábricas de termómetros, refinerías, clínicas dentales y en la minería y el procesamiento del oro y la plata extraídos con mercurio (OMS, 2013).

\section{Emisiones por lámparas fluorescentes y otras fuentes antropogénicas}

En un informe preparado para la EPA por RTI de la Oficina de Desechos Sólidos, titulado "Lámparas fluorescentes: evaluación preliminar de riesgo", se estima que aproximadamente 600 millones de lámparas se disponen cada año en EE. UU. Actualmente, la mayor parte de las lámparas están dispuestas de manera inadecuada; el $82 \%$ se deposita en vertederos; el $16 \%$ son incineradas; y solo el $2 \%$ son recicladas, según estimaciones (EPA, 1994).

En 1980, la EPA determinó que el mercurio puede migrar desde un vertedero de residuos sólidos municipales, en concentraciones significativas en el lixiviado, hasta alcanzar los acuíferos proveedores de agua de consumo humano (Brugnoni \& Iribarne, 2006). 
Tabla 1.

Fuentes de emisión de mercurio (INECC, 2000; EPA, 1997)

\begin{tabular}{l}
\hline \multicolumn{1}{c}{ Fuentes de mercurio } \\
\hline - Fundidoras secundarias de plomo \\
- Minería del oro y refinación \\
- Odontología (amalgamas) \\
- Refinerías de crudo \\
- Manufactura de cemento Portland \\
- Plantas de cemento \\
- Lámparas fluorescentes \\
- Termómetros \\
- Plantas termoeléctricas \\
- Fundidoras de hierro \\
- Cremación \\
- Baterías de mercurio \\
- Incineradores de residuos peligrosos biológico-infecciosos \\
- Residuos hospitalarios \\
- Esfignomanómetros \\
- Incineradores de residuos industriales peligrosos \\
- Gas natural \\
- Plantas carboeléctricas \\
- Combustión de la madera \\
- Producción de vidrio \\
\end{tabular}

\section{Efectos graves de la exposición al mercurio}

El pescado y el marisco son una parte importante de una dieta saludable. Sin embargo, casi todos estos contienen algún rastro de mercurio. El mercurio puede perjudicar especialmente a los fetos o el sistema nervioso en desarrollo de un niño pequeño. Los riesgos del mercurio en el pescado y el resto de mariscos dependen de la cantidad que se consuma y de los niveles del mercurio en estos (EPA \& FDA, 2004).

Un ejemplo de contaminación por compuestos orgánicos de mercurio sucedió en Minamata, Japón, entre 1932 y 1968. Fue el primer caso bien documentado; sucedió cuando se vertieron en la bahía de Minamata unas 27 toneladas de compuestos de mercurio y otros contaminantes. Esta ciudad está ubicada en la prefectura de Kumamoto, en la isla de Kyushu, Japón sudoccidental. Se vertieron en esa bahía subproductos orgánicos de mercurio, que eran el resultado de la producción de acetaldehído, cuando la empresa Chisso Corporation comenzó a desarrollar plásticos y perfumes (Yacuzzi, 2008).

Por lo expuesto en el párrafo anterior, se puede ver el mercurio y sus compuestos son sumamente tóxicos, especialmente para el sistema nervioso en desarrollo. El nivel de toxicidad en seres humanos y otros organismos varía según la forma química, la cantidad, la vía de exposición y la vulnerabilidad de la persona expuesta (Barbosa, De Sousa, Do Rea, Jardim, \& Fadini, 2003).

El metilmercurio tiene efectos adversos para muchos organismos vivientes. Algunos estudios indican que los pequeños aumentos en la exposición al metilmercurio pueden afectar negativamente al sistema cardiovascular (Montenegro \& Nicolalde, 2012). En otro estudio se demostró que a través de experimentos con ratas, expuestas a un 
compuesto inorgánico de mercurio (cloruro de mercurio), la necrosis tubular aguda (NTA, células renales dañadas por un tóxico o una sustancia dañina) por cloruro de mercurio (Hernández-Pando et al., 1995).

\section{Marco de referencia de seguridad química}

La ACGIH es una asociación científica, no es un órgano de implementación de normas. Como una organización científica, ha formado comités que revisan lo publicado por expertos. La literatura científica de la Conferencia Americana de Higienistas Industriales Gubernamentales (ACGIH, siglas en inglés) publica directrices conocidas como valores límite umbral (TLV) e índices de exposición biológica (BEI) para ser usadas por los higienistas industriales en la toma de decisiones con respecto a los niveles de seguridad en la exposición a diversos agentes químicos y físicos que se encuentra en el lugar de trabajo (ACGIH, 2012).

\section{Método}

\section{Tipo de estudio}

Estudio empírico con metodología cuantitativa; y descriptivo, mediante un código arbitrario de observación tipo estructurada. La investigación se lleva a cabo en el contexto habitual en el que se produce el fenómeno, pero el investigador introduce modificaciones para maximizar la probabilidad de aparición de dicho fenómeno (Montero \& León, 2007).

\section{Instrumentos utilizados}

\section{Dräger $X$-act 5000}

Bomba automática para tubos Dräger: proporciona las características de flujo requeridas por los tubos de rango corto Dräger; ofrece la opción de ser utilizada con tubos y sistemas de muestreo que requieren un flujo constante. La bomba automática Dräger X-act 5000 dirige fácilmente el aire que se debe medir a través de los tubos Dräger apropiados (Dräger, 2014).

\section{Máscara 3M 7800-S}

Los respiradores 3M de la serie 7800 están diseñados para usarse con los filtros de la serie 2000 y los cartuchos de las series 6000 y 7000 de 3M, proporcionando un alto nivel de protección en usos industriales Las principales aplicaciones para estos respiradores son en las operaciones de soldadura, en las industrias del aluminio, acero, y vidrio; en la farmacéutica, los agroquímicos, la minería; las industrias alimenticia, petroquímica y química, entre otras.

\section{Filtros 3 M 60926}

El filtro de carbón activado en base de azufre, que retiene mercurio en forma de amalgama, también es considerado como un desecho del proceso de tratamiento.

\section{Tubos de rango corto Dräger}

El tubo colorimétrico para vapor de mercurio $\mathrm{CH} 23101$ está certificado con ISO 9001 (certificado de manufactura); tiene un rango de medición desde 0.05 hasta $2 \mathrm{mg} / \mathrm{m}^{3}$. Los tubos son frascos de vidrio llenos de un reactivo químico que reacciona a una sustancia química o a una familia de productos químicos específicos. Un calibrado de $100 \mathrm{ml}$ de la muestra de aire es aspirado a través del tubo con la bomba automática Dräger. Si el(los) productos(s) químico(s) específico(s) está(n) presente(s), el reactivo en el tubo cambia de color y la longitud de ese cambio indica típicamente la concentración medida. Más de 500 sustancias diferentes pueden ser medidas con los tubos de rango corto Dräger (Dräger, 2011). Dräger X-act 5000.

\section{Unidades de análisis}

Se evaluaron 32 lugares que son edificios que contienen bastantes luminarias, ubicados en cinco departamentos de El Salvador, esto con el apoyo de accesibilidad que dio a los lugares que se deberían muestrear la Sección de Seguridad Ocupacional del Ministerio de Trabajo de El Salvador.

\section{Resultados de medición de mercurio en lugares donde hay lámparas en desuso o rotas}

Los resultados del muestreo de campo se contrastan con $0.025 \mathrm{mg} / \mathrm{m} 3$ como TLV de la ACGIH (como TWA [Tiempo Promedio Ponderado] piel A4). 
Tabla 2.

Resultados de visita con tres distintos sitios analizados

\begin{tabular}{cccccccc}
\hline Muestra N & Lámpara & $\begin{array}{c}\text { Embolada de } \\
100 \mathrm{ml}\end{array}$ & ug/l & $\mathrm{ppm}$ & $\begin{array}{c}\text { Desviación } \\
\text { estándar } \\
+--30 \%\end{array}$ & $\begin{array}{c}\text { TLV } \\
\mathbf{0 . 0 2 5} \\
\text { ug/l }\end{array}$ & Condición \\
\hline & & & & & & & \\
1 & Fluorescente & 40 (no reacción) & 0 & 0 & 0 & 0 & No peligro \\
2 & Fluorescente & 2 (reacción) & 1 & 0.12 & 0.3 & 40 & Peligro \\
3 & Fluorescente & 20 (reacción) & 0.1 & 0.012 & 0.03 & 4 & Peligro \\
\hline
\end{tabular}

En la tabla 2 se muestran los resultados obtenidos en las mediciones hechas en este lugar; por su extensión, se tomaron tres muestras en tres distintos sitios. En la primera muestra, bajo condiciones de $30{ }^{\circ} \mathrm{C}$ y $72 \%$ de humedad relativa, el tubo Dräger no tuvo reacción. En la segunda muestra con el tubo colorimétrico para medir mercurio la reacción fue en la carrera 2 , superando en cuarenta veces el TLV. Cabe destacar que en este sitio se encontraba una importante cantidad de lámparas rotas. En la tercera medición bajo las mismas condiciones el tubo Dräger reaccionó en la carrera 20, superando el límite cuatro veces de lo que establece ACGIH.

La imagen de la figura 4 corresponde al sitio donde se tomó la primera muestra; acá se encontró una cantidad menor a 40 luminarias rotas que ya tenían varias semanas de estar a la intemperie, expuestas al sol y a la lluvia.
La figura 5 corresponde al segundo sitio muestreado. En este lugar se encontraron los residuos de más de 500 lámparas rotas; la emisión de mercurio en el sitio fue la de mayor concentración encontrada en toda la investigación.

La imagen de la figura 4 corresponde al sitio donde se tomó la primera muestra, acá se encontraron una cantidad menor a 40 luminarias rotas que ya tenían varias semanas de estar a la intemperie expuestas al sol y a la lluvia.

La figura 5 corresponde al segundo sitio muestreado, en este lugar se encontraron los residuos de más de 500 lámparas rotas; la emisión de mercurio en sitio fue la de mayor concentración encontrada en toda la investigación. 
Figura 4.

Muestra 1. Foto propia.

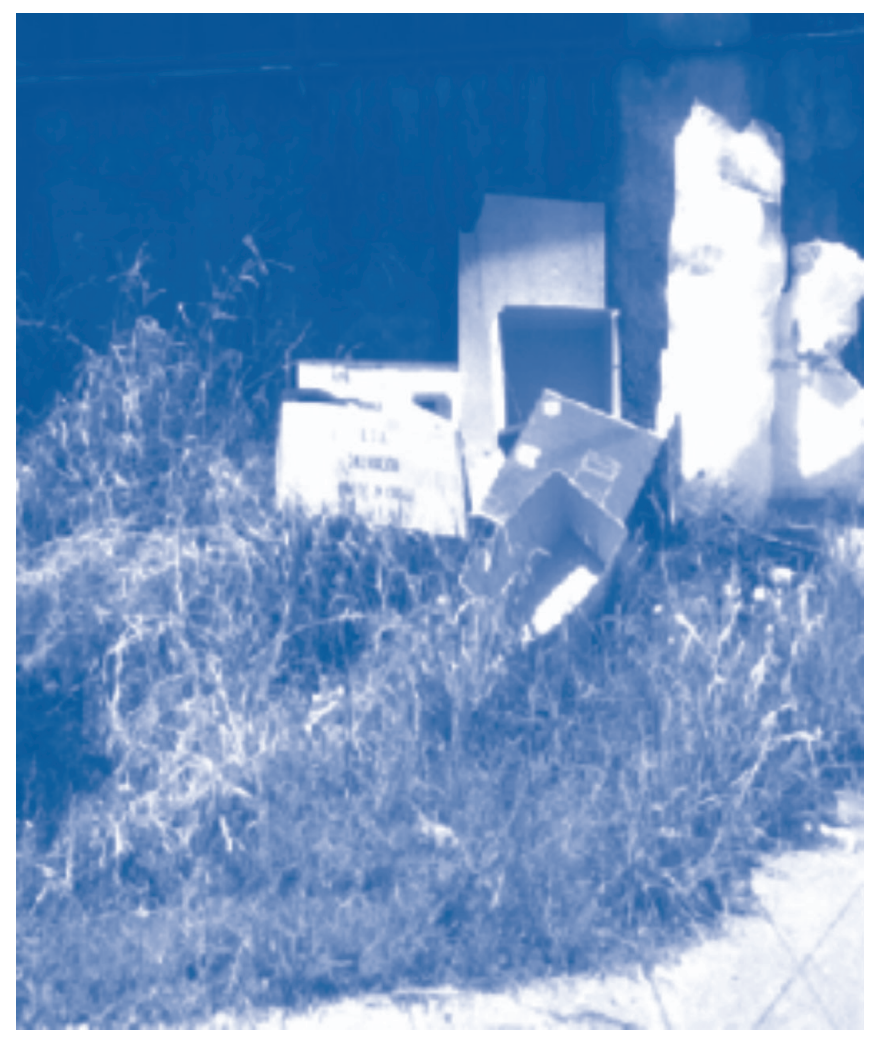

Figura 5.

Muestra 2. Foto propia.

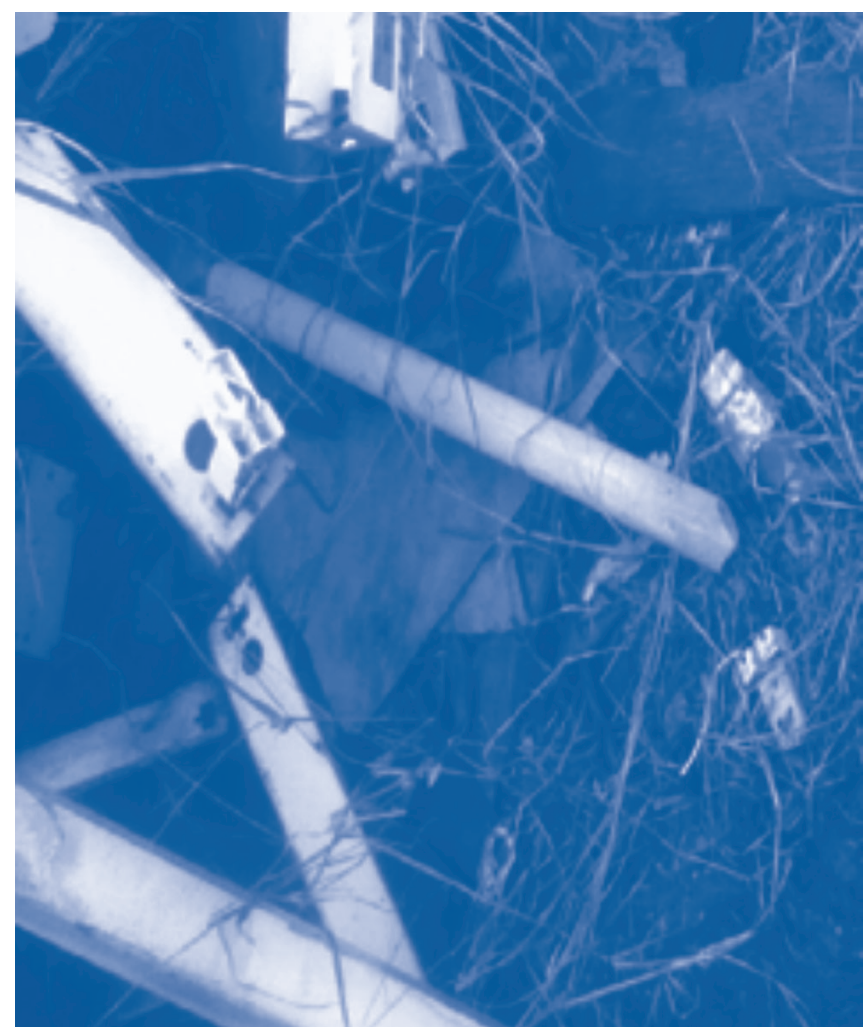

Tabla 3.

Resultados de visita con dos sitios analizados

\begin{tabular}{cccccccc}
\hline $\begin{array}{c}\text { Muestra } \\
\mathbf{N}^{\circ}\end{array}$ & Lámpara & Embolada $100 \mathrm{ml}$ & $\begin{array}{c}\text { ug/l de } \\
\mathrm{Hg}\end{array}$ & $\mathrm{ppm}$ & $\begin{array}{c}\text { Desviación } \\
\text { estándar } \\
+-30 \%\end{array}$ & $\begin{array}{c}\text { TLV } 0.025 \\
\text { ug/l }\end{array}$ & Condición \\
\hline & Fluorescente & 40 (reacción) & 0.05 & 0.006 & 0.015 & 2 & Peligro \\
5 & Fluorescente & 40 (no reacción) & 0 & 0 & 0 & 0 & No peligro \\
\hline
\end{tabular}

En la tabla 3 se muestran los resultados de la visita que se realizó a dos lugares, tomado muestras en cada uno de ellos. En el primero se tomó la muestra de tubos rotos que estaban a la intemperie con $29{ }^{\circ} \mathrm{C}$ y $56 \%$ de humedad relativa. El tubo Dräger reaccionó en la carrera 40, sobrepa- sando el límite de la del TLV. En la segunda medición, con temperatura de $28{ }^{\circ} \mathrm{C}$ y $56 \%$ de humedad relativa, después de cuarenta carreras de la bomba Dräger, el tubo de medición de mercurio no presentó reacción química. 
Figura 6.

Muestra 4. Foto propia

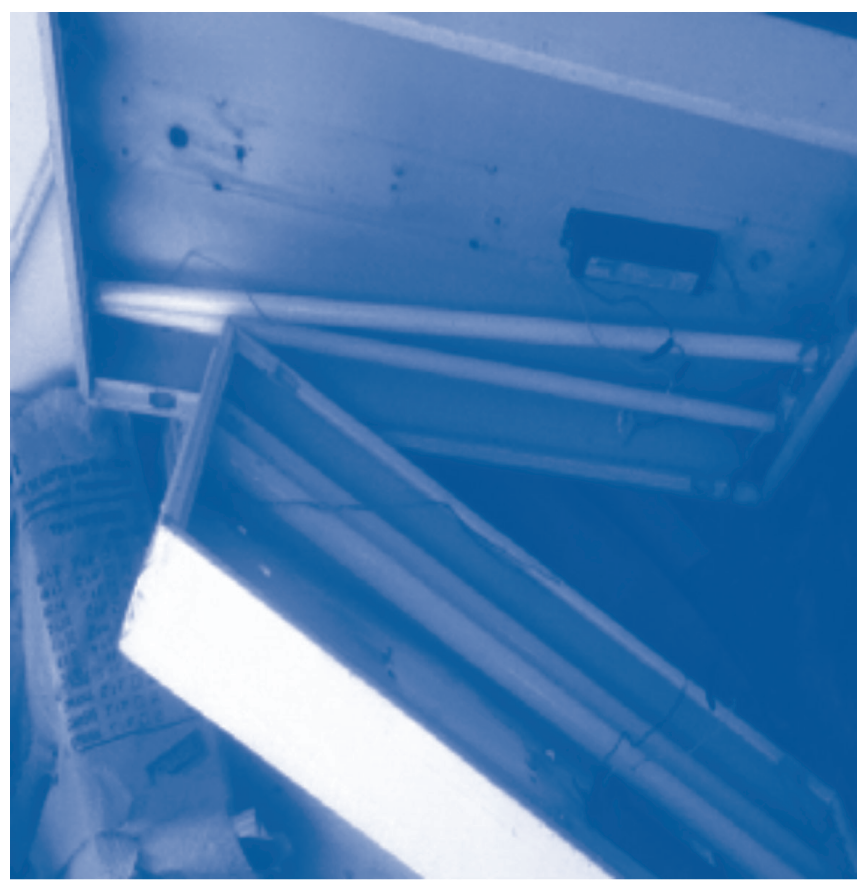

En la figura 6 se presenta el lugar donde se tomó la muestra 4; estas lámparas no estaban rotas pero presentaron emisiones hacia la atmósfera en el orden de $0.05 \mathrm{mg} / \mathrm{m}^{3}$, según reacción en tubo colorimétrico en la carrera 40 de la bomba Dräger.

Tabla 4.

Resultados de visita con dos sitios analizados.

\begin{tabular}{cccccccc}
\hline $\begin{array}{c}\text { Muestra } \\
\mathbf{N}^{\circ}\end{array}$ & Lámpara & Embolada $100 \mathrm{ml}$ & ug/l de $\mathrm{Hg}$ & $\mathrm{ppm}$ & $\begin{array}{c}\text { Desviación } \\
\text { estándar } \\
+-30 \%\end{array}$ & $\begin{array}{c}\text { TLV } \\
0.025 \\
\text { ug/l }\end{array}$ & Condición PA \\
\hline 6 & $\begin{array}{c}\text { Fluorescentes } \\
\text { enterradas } \\
\text { (\# no identificado) }\end{array}$ & 14 (reacción) & 0.14 & 0.0168 & 0.042 & 3.3 & Peligro \\
7 & $\begin{array}{c}\text { Fluorescentes } \\
\text { enterradas (40) }\end{array}$ & 9 (reacción) & 0.225 & 0.027 & 0.0675 & 9 & Peligro \\
\hline
\end{tabular}


En esta visita fueron dos los lugares inspeccionados: en el primero, las lámparas estaban enterradas. Se midió un grupo de lámparas T12 no determinado. El tubo Dräger reaccionó en la carrera 14 a $33.1{ }^{\circ} \mathrm{C}$ y $57 \%$ de humedad relativa, lo que indica que superó el TLV de 0.025 ug/l. En el segundo caso, las lámparas T12 enterradas eran alrededor de 40. La reacción del tubo indica que la emanación de vapor es mayor que la primera porque el tubo reaccionó en la carrera 9 a igual temperatura y humedad que en la medición anterior. En este caso, las lámparas enterradas pueden haberse roto. En ambos casos el mercurio está pasando a la tierra, al agua y a la atmósfera.

Figura 7.

Muestra 7. Foto propia

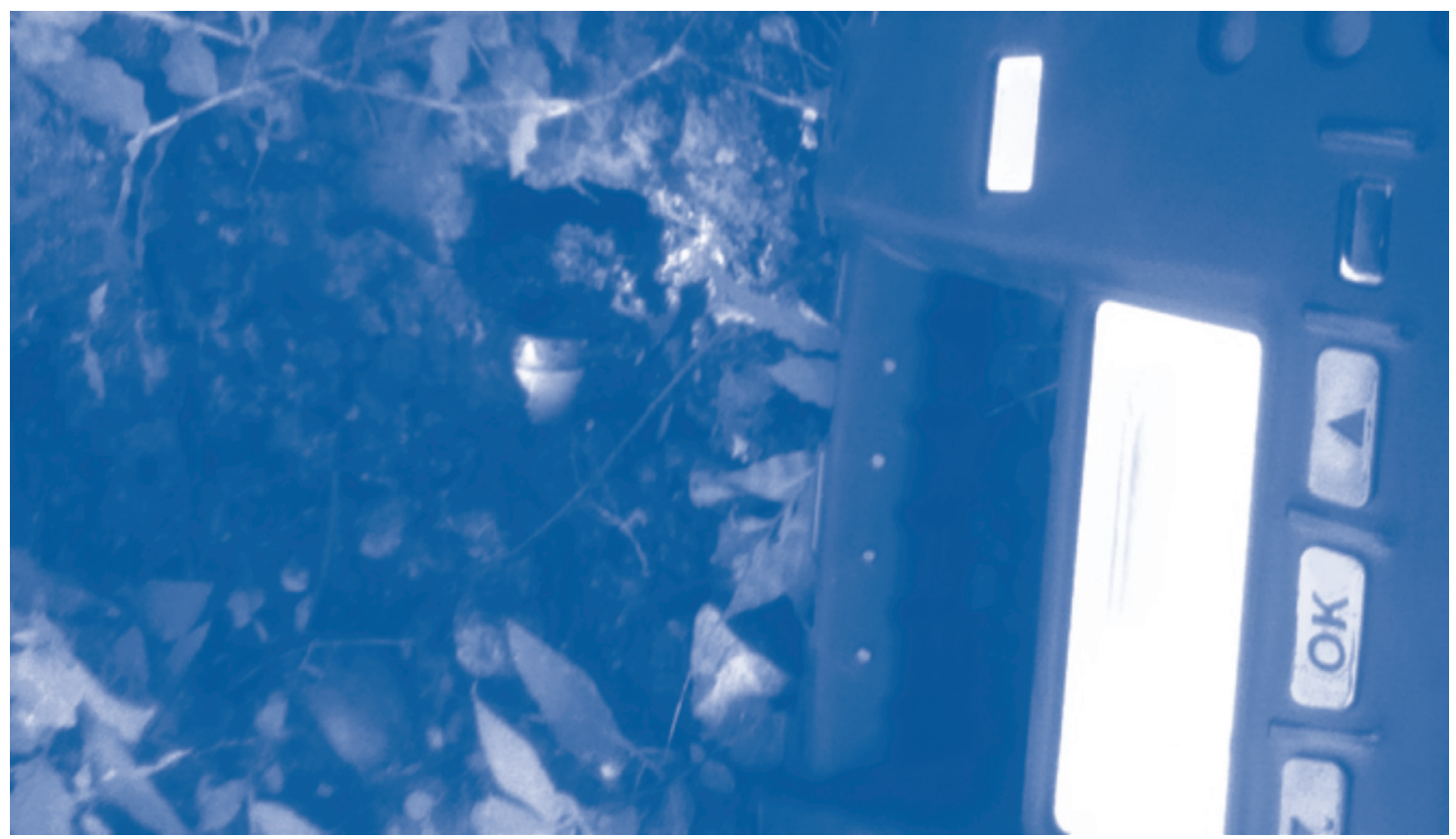

En la figura 7 se muestra la bomba automática Dräger en la medición de vapores de mercurio en lámparas. En esta medición las lámparas emitían vapores hacia la atmósfera; a pesar de estar enterradas, la máquina pudo determinar la concentración del vapor de mercurio.

\section{Conclusiones}

- En la mayoría de sitios visitados en esta investigación se encontraron restos de lámparas fluorescentes; y se halló que al medir con la analizador de gases había emanaciones de mercurio.

- Las lámparas fluorescentes en desuso en El Salvador contaminan la atmósfera, según las mediciones realizadas en el presente estudio, porque no existe un reglamento que regule una disposición final de este tipo de desecho pe- ligroso, pues contiene mercurio, elemento considerado por la OMS como una de las diez sustancias más tóxicas para el ser humano y para la vida.

- Los lugares donde se almacenan las lámparas en desuso deben de estar ubicados a 150 metros de las zonas habitadas, ya que se ha demostrado que no es necesario que estén rotas para que emitan emanaciones de mercurio a la atmósfera.

- Las lámparas fluorescentes en desuso y todos aquellos residuos que contienen mercurio son depositados en rellenos sanitarios; y cuando estos residuos son depositados, emiten gases de mercurio a la atmósfera; y también, cuando entran en contacto con los lixiviados, tienen la capacidad de llegar a los mantos freáticos, según los estudios encontrados relacionados con el tema del mercurio 
- Los medios de transporte de recolección de la basura son inadecuados para recoger lámparas que contienen mercurio, cuando estas son desechadas, ya que se demostró mediante mediciones hechas en el trabajo de campo que, al romperse, las lámparas fluorescentes emitían gases de mercurio, por lo que, debido a esto, los vehículos recolectores de la basura emiten mercurio en su camino hacia el relleno sanitario.

- No existen estudios en El Salvador sobre el grado de contaminación que hay en los rellenos sanitarios que reciben los desechos de todo el país. Se estima que las importaciones de lámparas fluorescentes a El Salvador tienen más de 74 años de realizarse; y nuca se ha dispuesto de forma adecuada de millones de lámparas fluorescentes lineales en desuso; y en años más recientes, también de lámparas fluorescente compactas, de mercurio y de alta presión. Todas estas son desechos peligrosos por su contenido de mercurio.

- En El Salvador no existe una planta de tratamiento para desechos que contengan mercurio (amalgamas dentales, lámparas fluorescentes, termómetros, barómetros, esfigmomanómetros, termostatos, etc.), según respuesta de la Oficina de Información y Respuesta del Ministerio de Medio Ambiente y Recursos Naturales.

\section{Referencias}

ACGIH. (2012). ACGIH Threshold Limit Values (TLVs) and Biological Exposure Indices (BEIS). Book. Copyright 2012.

Angulo, A.L.; \& Romero, R.M. (2006). "Análisis de alternativas para el manejo de lámparas fluorescentes de tubo desechadas". Bogota, D.C.: Universidad de la Salle, Facultad de Ingenieria Ambiental y Sanitaria. Recuperado de: http://repository.lasalle.edu.co/bitstream/ handle/10185/14104/00798194. pdf? sequence $=1$

Araujo, F.M. (2008). Curso de reacciones electroquimicas. Centro de equilibrios en solución. Universidad Central de Venezuela (UCV). P.12. Recuperado de:

http://www.ciens.ucv.ve/eqsol/Electroquimica/Clases/clases/Historia.pdf

Banco Central de Reserva de El Salvador, BCR, \& Ministerio de Hacienda-Direccion General de Aduanas (2014).
Importación de productos que contienen mercurio. Banco Central de Reserva, resolución de información RAIP N 042/2013 y Ministerio de Hacienda-Dirección General de Aduanas. IM-057868.

Barbosa, A.C., De Sousa, J.; Do Rea, J.G.; Jardim, F.W.; \& Fadini, P.S. (2003). "Mercury Biomagnification in a Tropical Black Water, Río Negro, Brazil. Brasilia: Environmental Contamination". Toxicologia. 45, 235-246.

Brugnoni, M.F. \& Iribarne, R. (2006). “Estudio de impactos en redes de distribución (I) y medio ambiente (II) debido al uso intensivo de lámparas fluorescentes compactas". Universidad de Buenos Aires. Recuperado de: http://energia3.mecon.gov.ar/contenidos/archivos/ Reorganizacion/eficiencia/Estudio\%20de\%20Impactos\%20en\%20Redes\%20de\%20Distrib\%20por\%20 LFCs.pdf

Cook, B. (1998). "High-Efficiency Lighting in Industry and Commercial Buildings". Originally published in: Power Engineering Journal, IEE, October 1998.

Constantinou, E.; Gerath, M.; Mitchell, D. \& Seigneur, C. (1995). "Mercury from power plants: a probabilistic approach to the evaluation of potential health risks". Kluwer Academic Publishers. Printed in the Netherlands. Water, Air, and Soil Pollution 80:1129-1138.

Dräger (2014). “Dräger X-act 5000, bomba automática para tubos Dräger. Lübeck, Alemania". Recuperado de: http://www.draeger.com/sites/assets/Publishinglmages/Products/cin_X-act_5000/US/9046996_PI_Xact_5000_EN_111113_fin.pdf

Dräger (2011). “Dräger-Tubes \& CMS Handbook. Lübeck. Soil, Water, and Air Investigations as well as Technical Gas Analysis". Publisher: Dräger Safety AG \& Co KGaA. Edición 16th.

Echazú, R., \& Cadena, C. (2012). “Medida en laboratorio de la emisión UV emitida por lámparas fluorescentes compactas", Salta, República de Argentina: Asades Avances en Energías Renovables y Medio Ambiente.

ELT (2006). "Lámparas fluorescentes". Zaragosa: ELT. Recuperado de: http://www.elt.es/documentos/dossier.pdf

EPA. (1997). "Locating and estimating air emissions from sources mercury and mercury compounds". EPA454/R-97-012.

EPA (1994). “Evaluación de mercurio en emisiones de lámparas fluorescentes rotas". Recuperado de: http://www. epa.gov/ttncatc1/dir1/mercury.txt 
Flavin D. (2008). Proyecto Marfa. ARQ (Santiago). Santiago Dic. $2008 \mathrm{n}^{\circ} 70$

General Electric. (2014). "Lámaparas fluorescentes". G.E. Recuperado de:

http://www.google.com.sv/url?url=http://www.guzman.cl/ catalogo/394/descargarCatalogo\&rct=j\&frm $=1 \& q=\& e$

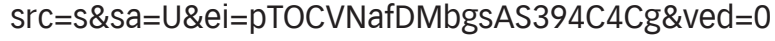
CB8QFjAFOA0\&usg=AFQjCNGM-ZTbIZfT4DhQ07fbuZ WXLIVF3Q

Hernádez-Pando, R.; Pedraza-Chaverri, J.; Orozco-Estévez, H.; Silva-Serna, P.; Moreno, I.; Rondán-Zárate, A.; Elinos, M.; Correa-Rotter, R. \& Larriva-Sahb, J. (1995). "Histological and subcellular distribution of 65 and $70 \mathrm{kD}$ heat shock proteins in experimental nephrotoxic injury". US National Library of Medicine, National Institutes of Health, Exp Toxicol Pathol. 1995 Dec.;47(6):501-8.

Indalux (2002). "Lámparas. Luminotecnia". Recuperado de:

http://www.ehu.es/alfredomartinezargote/tema_4_ archivos/luminotecnia/08.\%20Lamparas.pdf

INECC (2000). "Diagnóstico del mercurio en México". Recuperado de:

http://www.inecc.gob.mx/descargas/sqre/Diagnostico_hg_ mx_2002.pdf

INTI (2014). "Proyecto SAICM QSP: Mercurio en productos domésticos". Recuperado de: http://crsbasilea.inti. gov.ar/mer-lamparas.htm

Kayumov, L.; Lowe, A.; Rahman, S.C.; Casper, R. \& Shapiro, C. (2007). "Prevención de la supresión de la melatonina por la iluminación nocturna. Relevancia para el cáncer". European Journal of Cancer Prevention: August 2007 - Volume 16 - Issue 4 - pp 357-362.

Lamborg, Carl H.; Hammerschmidt, Chad R.; Bowman, Katlin L.; Swarr, Gretchen J.; Munson, Kathleen M.; Ohnemus, Daniel C.; Lam, Phoebe J.; Heimbürger, Lars-Eric; Rijkenberg, Micha J.A.; Mak A. Saito (2014). "A global ocean inventory of anthropogenic mercury based on water column measurements". Nature 512, 65-68, (07 August 2014).
Martínez F. (2003). Instalaciones eléctricas de alumbrado e industriales. Editorial Paraninfo

Montenegro, K.L., \& Nicolalde, A.S. (2012). “Diagnóstico y evaluación experimental del tratamiento de lámparas fluorescentes mediante el equipo Balcan modelo FSL 110 en Incinerox Cia. Ltda. Distrito metropolitano de Quito, 2012". Universidad Central de Ecuador, UCE. Recuperado de: www.dspace.uce.edu.ec/ bitstream/25000/490/1/T-UCE-0012-131.pdf

O'Donell, B.M.; Sandoval, J.D.; \& Paukste, F. (2006). “Capítulo 4. Fuentes luminosas". Lighting Handbook. Manual de Iluminación Eficiente. Editorial de la Universidad Tecnológica Nacional U.T.N. - Argentina. Buenos Aires 2006.

O’Neill, P. (1998). Enviromental chemistry. Published by Blackie Academic \& Professional, an Imprint of Thomson Science, printed in Great Britain by T. J. International, Padstow, Corwall. Third edition 1998

O'Neill, P. (1993). Enviromental chemistry. Chapman \& Hall. Printed in Great Britain, London. Second edition 1993.

OMS (2013). "El mercurio y la salud". Recuperado de: http:// www.who.int/mediacentre/factsheets/fs361/es/

PNUMA (2013). "El mercurio en productos y desechos. Nairobi". Recuperado de: http://www.unep.org/hazardoussubstances/Portals/9/Mercury/AwarenessPack/ Spanish/UNEP_Mod1_Spanish_Web.pdf

UGR (2013). "Química inorgánica ambiental. Metales pesados toxicos. El mercurio". UGR. Recuperado de: http://www.ugr.es/ mota/QIA_TEMA-3_Hg.pdf

Yacuzzi, E. (2008). "Chisso Corporation y la enfermedad de Minamata". Buenos Aires: Universidad del Cema. Recuperado de:

http://www.aotsargentina.org.ar/userfiles/CHISSO $\% 20$ CORPORATION\%20Y\%20LA\%20ENFERMEDAD\%20 DE\%20MINAMATA.pdf?PHPSESSID=15ec0e4788b9b8 1 fe4a07b6c0d9aae4d 